\title{
Investigation, Analysis and Evaluation on the Tourism Distributing Ability of Sanya City
}

\author{
Danhe Wang ${ }^{1, \text { a, }}$, Hui Gong ${ }^{1, \mathrm{~b}}$, and Zhao $\mathrm{Li}^{1, \mathrm{c}}$ \\ ${ }^{1}$ School of International Hospitality Management, Sanya University, Sanya 572022, China

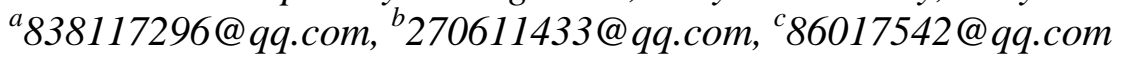

Keywords: Tourism distribution ability; Sanya city

Abstract: With the proposition and development of the tourism throughout Hainan Province, and the deep development and innovation of tourism resources in Hainan Province, more and more tourists to the island have chosen some tourism destinations except Sanya city, Haikou city and other traditional tourism destinations for the deep tourism experience. Therefore, the status and role of Sanya as a tourist distributing center begin to emerge gradually, and the tourism distributing ability of Sanya begins to attract more and more tourists. In the paper, the tourism distributing ability of Sanya is investigated and analyzed from three aspects including the internal traffic, the external traffic, and the agglomeration and diffusion of tourist flow. The urban space form, the urban road planning, the urban traffic site layout and the urban traffic management as well as the status of highways, railways, planes and liners in Sanya are investigated and analyzed. In addition, some characteristics of tourist flow in the process of agglomeration and diffusion are found. For example, Guangdong replaces the three traditional provinces of Northeast China became the first tourist source of Hainan Province during the investigation period. The Yangtze River Delta Region is transformed from a provincial capital tourist source to a small and medium-sized city tourist source. The agglomeration of tourist flow in Sanya is still dominated by the tourist source outside the province, but the tourist source in the province is developing gradually.

\section{Introduction}

Because of the geographical environment, the traffic condition and the distribution of tourism resources in Hainan Province, Sanya City has been taken as the first choice for tourism on the island in the early developing period of the tourism industry of Hainan Province. It is the most important tourism destination on Hainan Island. In recent years, Hainan Island has been promoted as an international tourism island. Lots of road resources such as the Island of Hainan Railway and the Expressway HAINAN have been developed and constructed. A series of measures, such as the deep development of tourism resources, have been taken in the eastern, western and central parts of Hainan Province. As a result, more and more tourists begin to choose some regions in the province except Sanya City for the deep tourism experience. Therefore, the status and role of Sanya City as a tourism distributing center begin to emerge. 
The tourism distributing ability of Sanya City is analyzed from three aspects including the internal traffic structure, the external traffic system and the agglomeration and diffusion of tourist flow. Besides, the internal traffic structure includes the urban space form, the urban road planning, the urban traffic site layout and the urban traffic management in Sanya City. The urban external traffic system mainly includes the land transportation (Highways and Railways), planes and liners. The agglomeration and diffusion of tourism flow mainly include the intensity and direction of distribution, the staying time, and the intensity and direction of diffusion.

\section{Investigation and Analysis of the Tourism Distributing Ability of Sanya City}

\subsection{Status of Traffic in Sanya}

\subsubsection{Urban Space Form}

The influence of the urban space on the distributing ability is that the urban topography is combined with the urban landform to form the geographical basis of the urban space. For example, plains are more beneficial to agglomeration than plateaus, and the river accumulation landform is more beneficial to agglomeration than the river erosion landform.

In Sanya City, it is high in the north and low in the south. It is surrounded by mountains in the north, and by the sea in the south. In "The Ecological Restoration City Repair Overall Planning in Sanya City", it is pointed out that the characteristics of "Tourist City" should be reflected in the overall space form of Sanya City.

The total area of Sanya City is 1919 square kilometers. In the topography of Sanya City, there are mountainous area with the proportion of $33.4 \%$, the hilly area with the proportion of $26.2 \%$, the moss marsh land with the proportion of $15.5 \%$, the valley with the proportion of $2.6 \%$ and the terrace plain with the proportion of $23.3 \%$. It is 91.6 kilometers long from east to west and 51.75 kilometers long from north to south

From east to west, there is a small lateral mountain system from Fuwanling to Huangling and finally to Yunmengshan Mountain, which separates the southern coastal hills, moss marsh land and plains from the northern mountains. In the south, it is divided into three parts by the two longitudinal mountain systems of Luhuiling - Tiananhoudaling and Haiyiling - Yalongling and Lizhiling- Taling. The whole city is divided into four blocks including the northern mountains, the eastern plains, the southern plains and hills, and the western hills and plains.

In Sanya City, there are the four districts of Yazhou District, Tianya District, Jiyang District and Haitang District (February 2014, the State Council approved the withdrawal of the six towns of Jiyang Town, Fenghuang Town, Haitangwan Town, Yacheng Town, Tianya Town and Yucai Town as well as the establishment of four new districts in Sanya City ) as well as some state-run farms such as Nantian Farm, Nanxin Farm, Licai Farm and Nanbin Farm. It covers an area of 1919.6 square kilometres.

According to the guiding principle of "The Overall Planning about Sanya City (2011 to 2020)", the construction level and international influence of the international tourism island shall be promoted, and the urban and rural planning, construction and management work shall be planned wholly in Sanya City. In 2020, the resident population shall be controlled within 500 thousand, the urban construction land shall be controlled within 74.7 square kilometers, and the population density shall reach 15 thousand people per square kilometer in the central urban area. The traffic pressure in the central urban area shall be relieved, which is conducive to the distribution of the large-scale tourist flow. 


\subsubsection{Urban Road Planning}

According to "The Overall Planning about Sanya City (2011 to 2020)", the low-carbon and environment-protection tourism traffic system shall be developed vigorously to construct a comprehensive traffic transportation system with the unique temperament of the coastal tourist city. The main trunk roads and the sub trunk roads shall be throughout the city, and the traffic transportation system of trams shall be planned in the coastal area.

This year, municipal roads are remodelled and constructed vigorously in Sanya City to form the backbone of the main trunk road network in the central urban area basically. However, it is still difficult for some main and sub trunk roads to withstand the test at the peak season of tourism and at the traffic peak. There are many patches of villages inside a city in the central urban area.

The main, secondary and branch systems can not be connected with each other so that tourists and citizens are dependant on the peripheral main trunk road network, which aggravates the traffic congestion on the main trunk road along the line.

In Sanya City, ten new road projects will be implemented in terms of road construction this year. It is planned to construct a total of $24.4 \mathrm{~km}$ of roads, including the Eastern-bank Mid-road in the Eastern-bank Squatter Settlement Area, the Eastern-bank Central Road, the Track West Road, the New Stretch of Lizhigou Road in Sanya City, the Upgrading and Transformation Project about the Road to Sanya University, the Municipal Road around Hongsen Hospital of Harbin Medical University, the New Stretch of Shuijiao Road in Sanya City, the Municipal Road around Hainan Tropical Ocean University, Huixin Road and the Connecting Line of Sanya Ring Highway. The total investment is about 138.88 billion yuan.

These road construction projects are combined with the squatter settlement project for the construction on main trunk roads, sub trunk roads and branch roads in blocks to improve the local traffic "micro-circulation" as well as the accessibility of the traffic network in the main urban area, which can alleviate the traffic pressure on the main road effectively and enhance the tourism distributing ability of the city.

\subsubsection{Urban Traffic Site Layout}

In "Sanya Tram Line Network Planning", it is mentioned that by 2020, four main tramways and two branch roads with a total length of $60.6 \mathrm{~km}$ will be built in Sanya City. In the period of "13th Five-Year", it is tried to achieve more than thirty percent of the urban bus sharing rate, to ensure that the average speed of motor vehicles in the central urban area at the peak hours is not less than twenty $\mathrm{km}$ per hour, and the average travel time of tourists and urban residents is within thirty minutes.

The four tramways are as followed:

T1: It is an urban backbone road with the functions of the urban life service, sightseeing and the collecting and dispatching transportation for an airport. It is from the Phoenix International Airport in Sanya City to Jiangang Road. Its branch is from Sanya Railway Station to Shengli Road.

T2: It is from Haiyue Square to Jiyang Town.

T3: It is from Haibo Holiday Resort to Haijiaotianya Scenic Area.

T4: It is an urban backbone road with the main functions of the the urban life service, sightseeing and the collecting and dispatching transportation for an airport. Its branch is aimed to provide the collecting and dispatching transportation service for the Cruise Home port of Phoenix Island.

In the aspect of inter-city passenger transportation, meet the requirement for the regional integration of the Integrated Tourism Economic Circle of "Great Sanya". Actively promote the construction on the inter-city bus system to realize the urban integration of inter-city passenger service in the Integrated Tourism Economic Circle of "Great Sanya". In the aspect of tourism 
passenger transportation, construct the tourism traffic network of the Integrated Tourism Economic Circle of "Great Sanya" with the integration among sea transportation, land transportation and air transportation. Specially open up cruise lines, HNHC SEABUS and the Boutique Tourism Route of "Great Sanya". Improve the public transportation system of rural tourism and the regional self-drive tourism system.

In the aspect of air passenger transportation, optimize the existing transportation organization mode and actively develop the service about the "Combination between Air Transportation and Rail Transportation" with Haikou Meilan International Airport to expand the service scope of the "Combination between Air Transportation and Rail Transportation".

\subsubsection{Urban Traffic Management}

The traffic civilization in Sanya City is greatly improved this year, but there are still many problems about the urban traffic civilization, mainly including the cutting-in and reckless driving behaviors of buses, taxis and cars, electric cars running on the eastern side of airports and motorized driveways, electric cars running a red light and retrograding, government cars running a red light, and passersby not obeying traffic rules.

At present, many departments in Sanya City improve the traffic order properly, strengthen the improvement on the traffic order, and make all efforts to create a city with good traffic. The Municipal Public Security Bureau officially launches the "Road Traffic Management Road Management System" in the whole city, insists on the general goal of "Ensuring Traffic, Promoting Safety and Providing Good Service", and adheres to the principle of " The Mobilization of All Police and the Participation of All People".

It specially sets up a leading group and the organizational structure of the road managers at all levels. All police classification resources are integrated to divides it into the First- grade "Lu Zhang", the Second-grade "Lu Zhang", the Third-grade "Lu Zhang" and the Road Manager according to jurisdictions and business functions. Implement the binding of responsibilities as well as the binding of duties. It is ensured that the public security organs can dispatch the police quickly and deal with it in time if emergency accidents occur.

At present, the effective integration and connection between the public passenger transportation mode and the mass tourist traffic are established in Sanya City. In addition, the effective transportation mode can be realized quickly among airports, railway passenger stations and long-distance passenger transportation centers so that tourists can get to a scenic area easily by using the special-line public traffic transportation mode. Within half a day, self-drive tourists can reach their destinations quickly within half a day.

\subsection{Status of the External Traffic in Sanya}

Sanya City is located in the southern part of Hainan Island. At present, the external transportation mode is divided into highways, railways, planes and liners in Sanya City.

\subsubsection{Highways and Railways}

In a region, the development scale and space of the tourism industry are greatly determined by the monopoly tourism resources and the linked tourist channels according to the distribution tourism flow direction. Because of the other regions of Hainan Province with poor accessibility as well as Sanya City with relatively rich tourism resources, many modes of transformation in Hainan Province such as railways, highways, planes and liners are mostly centered as Sanya City, resulting in a kind of divergent traffic network. 
The trunk railway lines currently operated in Sanya City include Yue-Hai Railway accelerated and transformed in 2007, the Eastern Island of Hainan Railway opened at the end of 2010, and the Western Island of Hainan Railway opened at the end of 2015. The three trunk railway lines were introduced into Sanya City from the east and the west respectively, and centered as Sanya Station.

The tourism traffic in Hainan Province is dominated by the Island of Hainan Railway and the Expressway HAINAN. There are the Eastern Island of Hainan Railway, the Western Island of Hainan Railway, and Expressway HAINAN in the whole province. They are all centered as Sanya City and Haikou City and supplemented by national highways to radiate the whole province.

Its unique annular design is connected effectively through stations as well as some traffic transportation modes such as highways, international airports and railway car ferries to construct a three-dimensional traffic system to meet the diversified travel demand of people. Visitors can reach any scenic area of the province from Sanya City within half a day by high speed rail or car. It greatly guarantees the accessibility of the main scenic areas and tourism attractions.

\subsubsection{Civil Aviation}

In 2016, the passenger throughput of the Phoenix International Airport in Sanya City was 17 million and 370 thousand, with a growth rate of $7.3 \%$. Thereinto, there were 8 million and 690 thousand person-times with a growth rate of $6.9 \%$. There were 112771 air schedules with a growth rate of 5.5\%. The transport turnover amount and thr passenger throughput were shown in Table 1.

In order to meet new challenges, it is planned to build a new airport in Sanya City. Hongtang Bay is taken as the first site of the airport. It is planned to fill in the sea with an area of 28.18 square kilometers to build a sea airport about four kilometers away from the shore and about thirty-five kilometers from the urban center. It is estimated that the construction on the main body and supporting engineering of the airport will be completed by December 31st, 2020, and the trial operation will be started on December 31st, 2020.

It is designed that the annual passenger throughput of the new airport is 60 million. It will become a regional international hub airport and free trade and cooperative development platform facing ASEAN and even Asia Pacific and Africa. The improvement of aviation conditions will greatly improve the local business and tourism market to enhance the comprehensive regional economy.

\subsubsection{Liner}

Phoenix Island International Liner Port was built on November 9th, 2006. A dock with 100 thousand tons of passenger throughput was put into use. It is the only international liner port in Sanya City. It is the gateway of international tourists to Sanya City. At present, in the second-phase project, a dock with 30 thousand tons of passenger throughput, a dock with 100 thousand tons of passenger throughput, two docks each with 1150 thousand tons of passenger throughput and a dock with 2250 thousand tons of passenger throughput are constructed, resulting in a tourism reception capacity of 2 million person-times per year. On the island, there is a modern passenger transport joint inspection building with five frontier inspection channels covering an area of nearly ten thousand square meters. Three thousand international tourists can be received at once.

\subsection{Agglomeration and Diffusion of Tourist Flow}

\subsubsection{Investigation and Research on the Distribution of Tourist Flow in Sanya}

In order to enrich and supplement the research content, a field survey was carried out in the research in order to realize the mutual verification and echo between the first-hand information and 
the second-hand information. It was conductive to getting more detailed and more objective data to enrich the research results ultimately.

\subsubsection{Investigation Design and Investigation Process}

In the research, tourists to Sanya were taken as the research object for the sample survey. It was developed in the questionnaire survey way among tourists and the interview way among the tour operators from travel agencies from December 2017 to October 2017. It was in the peak period of tourism in Sanya City. The sample was based on individual tourists.

A total of two hundred questionnaires were distributed and all of them were recovered, resulting in an effective rate of a hundred percent. At the interview stage, respondents included tourists and the managers from travel agencies. It was conductive to getting more objective and more real results through the combination between the quantitative analysis and qualitative analysis of questionnaires.

In addition, in order to obtain more objective and more real survey data, the choice of investigation sites should be in line with the requirement for variety and complementarity. Scenic areas (Sanya Bay, Big East China Sea and Yalong Bay) and hotels were chosen mainly. The tourists to scenic areas endowed with strong representativeness and real results were conductive to the analysis of data. Hotels were comfortable, which was conductive to the communication and exchange visits among visitors.

\subsubsection{Application of Likert Scale}

In the survey, in order to facilitate expression and analysis with the quantitative results, some views about impressions were quantified through Likert Scale as much as possible. It was put forward by American social psychologist Likert on the basis of the improvement on the original summated rating scales in 1932. It is widely used in the process of social investigation.

Likert Scale can be adopted to measure the average intention of a group on something. It is usually designed as a series of questions and expressions related to the survey purpose, so that respondents can be selected within the scope of the existing options. In design, the five-level comments are often used. Finally, some corresponding mathematical methods are adopted to obtain data for the quantitative analysis.

\subsubsection{Analysis of Investigation Results}

1) Analysis of the Situation about the Agglomeration of Tourist Flow in Sanya City

Mode of Transformation, Age Structure and Consumption in Sanya

In the sample survey among tourists, forty-three percent of the respondents went directly to Haikou City and the rest respondents went directly to Sanya City, which showed that Sanya was still the first tourism destination and tourism distributing center in Hainan Province. In terms of the mode of transportation, eighty-seven percent of the respondents arrived in Sanya by plane. In the survey, the visitors to Sanya were endowed with an obvious young characteristic.

In addition, the family travel and the high-end visitors became the mainstream. According to statistics, young and middle-aged tourists with strong spending power and plentiful physical strength account for $56.17 \%$. The family-based tourists account for $44.66 \%$ of all the tourists. The sample survey showed that the tourists with the family monthly income of more than eight thousand yuan accounted for $57.91 \%$. Those with the family monthly income of more than twelve thousand yuan accounted for $22.08 \%$. Moreover, the average expenditure of the seventy-seven percent of the visitors to Sanya City was two thousand yuan per day. 
The survey data showed that in the whole trip to Hainan Province, the average expenditure of tourist in the process of going shopping in Sanya City was 1039 yuan, accounting for twenty-two percent of the total travel expenditure. The tourism products tourists liked buying best included speciality products, Yellow Pear handicrafts and special souvenirs.

Number and Direction of Domestic Tourists

Hainan Province is located at the southernmost end of China, so the most domestic tourists are from North China. In the survey, the six main tourist sources (Provinces/Cities) included Guangdong Province, Beijing City, Shanghai City, Jiangsu Province, Zhejiang Province, Sichuan Province and Hainan Province. And there were some characteristics as followed:

(1) Guangdong replaced the Northeast Three Provinces as the first tourist source during the survey period in Hainan Province. The tourists from Guangdong Province, a province with the similar geographical advantage of Hainan Province, had an enormous advantage in both the tourism cost and the time cost, which made Sanya City an important tourism destination for Cantonese.

(2) The Yangtze River Delta was transformed from the provincial capital tourist source to the small and medium-sized city tourist source. The survey showed that the tourist source of the region had been transformed from the original provincial capital or the first-line city to the non provincial capital. The number of the tourists from Ningbo City, Suzhou City, Wuxi City, Wenzhou City and other medium-sized cities had exceeded that from traditional municipalities and provincial capitals such as Shanghai, Nanjing and Hangzhou.

(3) The agglomeration of tourist flow in Sanya City was still dominated by the tourists from other provinces, but the number of the tourists from the province was growing constantly.

The average time of tourists to stay in Sanya

According to the survey, the average time of visitors to reserve apartments and residential accommodation was 7.7 days. They time to stay in Sanya City was longer than that in other destinations. Moreover, there were more family-based visitors.

For this kind of tourists, one the one hand, as the staying time got longer, the price of traditional hotels could be increased. On the other hand, it could not meet the travel experience in family gatherings. However, apartments and residential accommodation could meet the need such as the equipped matching kitchens, washing machines and multi-bedroom suites. In addition, being different from hotels which were constructed centrally, apartments and residential accommodation were distributed in various communities in Sanya City. With higher coverage, it was more convenient for the choice of tourists, so that visitors could also better experience the local cultural life and appreciate the more beautiful scenery.

2) Analysis of Diffusion after the Agglomeration of Tourist Flow into Sanya

(1) The mode of diffusion was mainly based on the short and multi-purpose tourism. Because of the unique geographical location limit of Hainan Province, there were only international airports in Haikou City and Sanya City in Hainan Province. As a result, for tourists, if they wanted to travel to other cities in Hainan, they must take Haikou City or Sanya City as a tourism distributing center.

(2)The high-level scenic area was taken as the baton and vane of the diffusion of tourist flow. The fact that tourists entered Sanya City belonged to the typical long-distance and large-scale tourism space behavior characteristics. In the long- distance and large-scale tourist behavior, tourists tended to take the high-level scenic area as a tourism destination. Most of the 5A-level scenic areas in Hainan Province were concentrated in Sanya City and its surrounding areas. Therefore, the tourist flow to Sanya for the first time could also be concentrated in Sanya and its surrounding areas. The survey showed that for tourists who had been to Sanya City, their travel direction was mainly concentrated in the other 4A or 3A scenic areas as well as their surrounding areas in Hainan Province. Moreover, it was focused on the deep experience tour. 


\section{Conclusion}

When the tourism competition is no longer the competition among simple resource points, the comparative advantage of the tourism industry is no longer the advantage formed by the simple tourism resources, and the competition of regional tourism is also endowed with more elements and connotation.

The tourism distributing center is a new geographical thing that appears with the development of regional tourism. The tourism distributing center is responsible for the agglomeration and divergence of tourist flow, capital flow, material flow and information flow. In the paper, the function of the tourism distributing center in Sanya City is taken as the research object to draw the following conclusions:

(1) Make rational use of the urban space form, and improve the planning and construction on transportation infrastructures in Sanya City. Further improve some traffic transportation infrastructure such as highways, railways, aviation and ports, improve the traffic transportation condition in the city and the surrounding area, and strengthen the internal and external traffic connections in the city. Establish a multi-level and multi-type comprehensive urban transport system with the public transportation as the main body and various modes of transportation to facilitate the transfer of different modes of transportation. Carry out the planning and layout on parking lots to promote the construction on urban parking lots.

(2) Deeply dig the great value of the tourist distribution center so as to enhance the efficiency of the local tourism industry in Sanya City.Fully highlight the geographical advantage, the traffic advantage and the industrial advantage, further strengthen the function of the tourism distributing center and promote the development of the tourism industry through the promotion on the distributing function in the city.The unique traffic condition, the status of the tourism center, the international service industry development status and the unparalleled climate condition of Sanya City are incomparable in Hainan Province. In Sanya City, continue highlighting the status of the tourism center, give full play to the advantages of location and traffic, further excavate the function of the tourism distributing ability, and promote the development of the local tourism industry through the distributing function.

\section{Acknowledgments}

This work was financially supported by Social Science Foundation fund of Hainan Province.(Grant No. HNSK (ZC) 17-23) and Social Science Foundation fund of Sanya City (Grant No. SYSK17-21)

\section{References}

[1] Goh; Li; Li; A Comparative Analysis of Domestic and International Tourism Spatial Distribution: Trends and Impacts, Routledge. 2014, 388-413.

[2] Information on http://www.sanyatour.com. 